\title{
Excitation of the symmetry forbidden bending mode in molecular photoionization
}

\author{
J. Scott Miller ${ }^{a}$ \\ Department of Chemistry, Louisiana State University, Baton Rouge, Louisiana 70803 \\ E. D. Poliakoff ${ }^{b)}$ \\ Department of Chemistry and Department of Physics and Astronomy, Louisiana State University, \\ Baton Rouge, Louisiana 70803
}

Thomas F. Miller III, Alexandra P. P. Natalense, ${ }^{\mathrm{c})}$ and Robert R. Lucchese Department of Chemistry, Texas A\&M University, College Station, Texas 77843

(Received 6 October 2000; accepted 19 December 2000)

\begin{abstract}
We present results on the energy dependence of the vibrational branching ratio for the bending mode in $\mathrm{CO}_{2} 3 \sigma_{u}^{-1}$ photoionization. Specifically, we determine the $v^{+}=(0,1,0) / v^{+}=(0,0,0)$ intensity ratio by detecting dispersed fluorescence from the electronically excited photoions. The results exhibit large deviations over a very wide energy range, $18<h \nu_{\text {exc }}<190 \mathrm{eV}$. Production of the $v^{+}=(0,1,0)$ level of the ion from the $v_{0}=(0,0,0)$ ground state is forbidden by symmetry, and while observations of such features are well established in photoelectron spectroscopy, their appearance is normally ascribed to vibronic coupling in the ionic hole state. In this case, we find that such explanations fail to account for the energy dependence of the branching ratio. These deviations indicate that the continuum photoelectron participates in transferring oscillator strength to the nominally forbidden vibrational transition. A theoretical framework is developed for interpreting the experimental data, and Schwinger variational calculations are performed. These calculations demonstrate that the continuum electron is responsible for the observation of the excited bending mode as well as its energy dependence. This is an intrachannel effect that is best described as photoelectron-induced vibronic symmetry breaking. This appears to be a general phenomenon, and it may be useful in illuminating connections between bond angle and photoionization spectroscopies. The magnitude of these deviations display the utility of vibrationally resolved studies, and the extent over which these changes occur underscores the necessity of broad range studies to elucidate slowly varying characteristics in photoionization continua. (C) 2001 American Institute of Physics. [DOI: 10.1063/1.1349090]
\end{abstract}

\section{INTRODUCTION}

The study of molecular photoionization provides an ideal opportunity to investigate how electronic and vibrational motion are correlated in fundamental scattering processes, and one specific observation that generates considerable interest is the occurrence of transitions that are nominally forbidden. One such forbidden transition is the creation of the ion with a single quantum of vibration in a nontotally symmetric mode, such as the bending vibration for a linear triatomic system. In this study, we report on the $v^{+}=(0,1,0) / v^{+}=(0,0,0)$ vibrational branching ratio following $3 \sigma_{u}^{-1}$ photoionization of $\mathrm{CO}_{2}$ for photon energies 18 $\leqslant h \nu_{\text {exc }} \leqslant 190 \mathrm{eV}$. This is done by detecting dispersed fluorescence from electronically excited photoions. In this paper we expand on an initial report of this work. ${ }^{1}$ In the previous

\footnotetext{
a) Current address: Air Force Research Laboratory/VSBS, Hanscom AFB, Massachusetts 01731-3010.

b) Author to whom correspondence should be addressed. Electronic mail: erwin.poliakoff@chem.lsu.edu

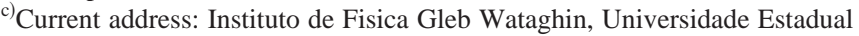
de Campinas, 13083-970 Campinas, SP, Brazil.
}

paper, we showed energy-dependent data, which suggested that the continuum electron plays an active role in the production of the $v^{+}=(0,1,0)$ level. In this paper, we present theory that corroborates the main conclusions from the preliminary study, and also elucidates the mechanism by which this symmetry forbidden transition is occurring. The theory suggests that the transition is a result of intrachannel coupling with the direct participation of the continuum electron. The analysis is bolstered by Schwinger variational calculations for the continuum photoelectron, ${ }^{2-4}$ and a key result is that semiquantitative agreement between experiment and theory is achieved without invoking intensity borrowing from alternative ion-hole states. To our knowledge, this is the first example where such an effect-i.e., photoelectroninduced vibronic symmetry breaking-has been used to explain either the existence or the relative intensities of nominally forbidden vibronic transitions. In clarifying the mechanism of this forbidden transition, we show that it can be exploited for probing previously hidden aspects of the photoionization scattering dynamics. In addition to our preliminary report on these findings, this is the first study to probe the energy dependence of vibrational branching ratios 
for the bending degree of freedom, and this energy dependence suggests useful applications in chemical physics, as well as in related fields.

For the present study, the experimental information about symmetry forbidden vibronic transitions is generated by ejecting electrons from an inner valence orbital of the target molecule, then monitoring the fluorescence spectra as the outer valence electron relaxes to fill the inner valence shell vacancy. The outer valence configuration of $\mathrm{CO}_{2}$ is $\ldots\left(4 \sigma_{g}\right)^{2}\left(3 \sigma_{u}\right)^{2}\left(1 \pi_{u}\right)^{4}\left(1 \pi_{g}\right)^{4}$, and the ionization threshold for $3 \sigma_{u}^{-1}$ photoionization in $\mathrm{CO}_{2}$ is $18.077 \mathrm{eV} .^{5}$ The excitation/fluorescence sequence is given by Eq. (1):

$$
\begin{aligned}
\mathrm{CO}_{2}[ & \left.X^{1} \Sigma_{u}^{+}, v_{0}=\left(0, v_{2}, 0\right)\right]+h \nu_{\mathrm{exc}} \\
\rightarrow & \mathrm{CO}_{2}^{+}\left[B^{2} \Sigma_{u}^{+}, v^{+}=\left(0, v_{2}^{+}, 0\right)\right]+e^{-}, \\
& \downarrow \\
& \mathrm{CO}_{2}^{+}\left[X^{2} \Pi_{g^{\prime}} v^{\prime \prime}=\left(0, v_{2}^{\prime \prime}, 0\right)\right]+h \nu_{\nu^{+} v^{\prime \prime}} .
\end{aligned}
$$

We are able to vary the experimental conditions to increase hot band ionization, i.e., $v_{2}=1$, or we can enhance the sequence with $v_{2}=0$ by lowering the temperature. A key point of the dispersed fluorescence measurements is that the detection bandwidth is decoupled from the excitation bandwidth. The advantage of this decoupling is that highly resolved photoion fluorescence spectra are accessible regardless of the bandwidth of the excitation source, ${ }^{6,7}$ as opposed to photoelectron spectroscopy, where the excitation bandwidth is the lower limit to the detection bandwidth. The disadvantage is that the "parentage" of a fluorescing level is not determined directly, but must be inferred from other evidence. For the present study, there are sufficient clues to infer the dominant production mechanism of the $v^{+}=(0,1,0)$ level based on the thermal dependence of the branching ratios.

Observations of bending excitation [i.e., $v_{0}=(0,0,0)$ $\left.\rightarrow v^{+}=(0,1,0)\right]$ are well documented via photoelectron spectroscopy. An early study was performed by Wannberg et $a{ }^{8}{ }^{8}$ This study used resonance lamps coupled with a photoelectron spectrometer. Wang et al. ${ }^{5}$ employed a supersonic expansion for a high resolution photoelectron spectroscopy study of $\mathrm{CO}_{2}$, which permitted the acquisition of vibrationally resolved spectra with higher quality data. However, all of these studies have been constrained to fixed energies, as resonance lamps were used as excitation sources. More recently, ZEKE-PFI spectroscopy has generated much more highly resolved data in studies of photoionization dynamics. Merkt et al. ${ }^{9}$ have used ZEKE-PFI spectroscopy to study the rotational substructure in bending excitation transitions in $\mathrm{CO}_{2}$, which is invaluable in discerning the propensity rules for angular momentum transfer accompanying photoionization. Moreover, bending transitions have been observed in synchrotron-based ZEKE-PFI studies of $\mathrm{CO}_{2}$ and its valence isoelectronic analogs. ${ }^{10-13}$ However, ZEKE-PFI cannot probe the evolution of the continuum photoelectron wave function because such studies, by definition, are limited to threshold.

The first attempt to elucidate the origin of a symmetry forbidden photoionization transition using energy-dependent data was a synchrotron radiation study by Roy et al. ${ }^{14}$ They determined the energy dependence asymmetry parameter for the forbidden $(1,0,1)$ level in $4 \sigma_{g}^{-1}$ photoionization of $\mathrm{CO}_{2}$. They ascertained that the origin of the forbidden band in the $C^{2} \Sigma_{g}^{+}$state photoelectron spectrum could be attributed to interchannel vibronic coupling to the $B^{2} \Sigma_{u}^{+}$state, based on how the asymmetry parameter for these two channels tracked each other as a function of energy in the range $20-28 \mathrm{eV}$. In the current paper, we will demonstrate that performing the studies over wider spectral ranges (i.e., $c a .200 \mathrm{eV}$ ) provides a revealing perspective on the process. Indeed, the utility of wide energy coverage for discerning the parentage of nominally forbidden transitions has been made in previous studies on the origin of satellite spectra in inner-valence photoionization by Karlsson, Holland, and co-workers. ${ }^{15-19}$ A primary goal of this study is to provide sufficient energydependent information so that it will be possible to identify the underlying cause for the production of the $v^{+}=(0,1,0)$ level.

Finally, we note that once the mechanism for production of the excited bending level is understood, it can be exploited in a variety of ways. Theoretical ${ }^{20}$ and experimental ${ }^{21}$ studies that revealed the connection between ionization dynamics and geometry have already generated strategies for probing local geometric information of simple adsorbates on solid surfaces. $^{22-25}$ In order to achieve comparable progress for complex molecular targets, vibrationally mode-specific investigations are essential. This is because such studies allow us to test the response of the ionization dynamics to alternative vibrational motions. However, mode-specific investigations are impossible if selection rules preclude the excitation of vibrations corresponding to the motions of interest. Thus it is necessary to understand how rigorous the selection rules are, and if they are not absolute, the mechanism by which they are being violated. While synchrotron-based molecular photoionization studies of forbidden transitions are uncommon (with notable exceptions ${ }^{14-19}$ ), the present study demonstrates that they are both feasible and desirable as they highlight new connections between photoionization dynamics and molecular geometry. Finally, the current study reinforces the need for wide spectral coverage, as deviations cover a broad range, similar to behavior observed in a previous investigation on diatomic photoionization. ${ }^{26}$

\section{EXPERIMENT}

The experimental method is described in detail elsewhere, ${ }^{7,27}$ so we focus on aspects that are pertinent to the present study. The apparatus is shown schematically in Fig. 1. Monochromatized synchrotron radiation intersected a beam of gas molecules emerging from a free jet expansion. Fluorescence from excited state ions was collected, dispersed, and detected. The synchrotron radiation was produced by the Louisiana State University Center for Advanced Microstructures and Devices (CAMD) facility ${ }^{28}$ with typical injection currents of $225 \mathrm{~mA}$. The radiation from the synchrotron was monochromatized by a plane grating monochromator ${ }^{29}$ operated in the high-throughput mode i.e., the premirror angle was optimized for flux. The slits of the 


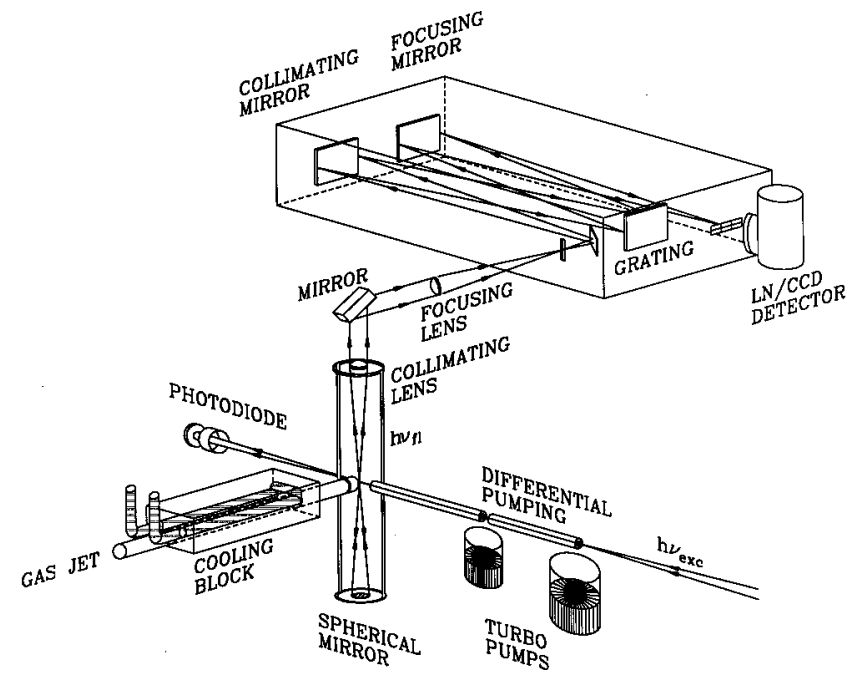

FIG. 1. Experimental schematic.

excitation monochromator were set at $2 \mathrm{~mm}$, resulting in a bandwidth varying between $0.1-0.5 \mathrm{eV}$ and an incident photon flux of $\sim 5 \times 10^{11} \mathrm{~h} \nu / \mathrm{s}$.

The ultrahigh vacuum of the beamline and electron storage ring were separated from the sample chamber by a twostage differential pumping apparatus. A $2 \mathrm{~mm}$ i.d. capillary intercepted the excitation photon beam at the focus of the refocusing mirror and served as a light pipe to direct the incident radiation to the sample. There was a gap in the capillary of approximately $3 \mathrm{~mm}$ that was pumped by a 60 $\mathrm{L} / \mathrm{s}$ turbomolecular pump, and the refocusing mirror section of the beamline was pumped by a $170 \mathrm{~L} / \mathrm{s}$ turbomolecular pump. We could adjust the gas throughput until the background pressure in the sample chamber reached $5 \times 10^{-4}$ Torr and still maintain the refocusing mirror chamber of the beamline at $P \approx 10^{-9}$ Torr. This effective differential pumping was essential, as the experiment required relatively high resolution on a weak transition.

It was necessary to control the vibrational population of the neutral target gas thermally in order to assess the contributions of hot-band ionization transitions. The apparatus is capable of achieving stagnation temperatures from 243 to $450 \mathrm{~K}$. For high-temperature studies, the gas sample was heated to approximately $423 \mathrm{~K}$ in the gas jet prior to expansion and introduced into the chamber as a supersonic free jet of the pure target gas. This achieved some rotational cooling while keeping the vibrational temperature elevated, yielding a sizable population of $v_{0}=(0,1,0)$ target molecules $(\sim 15 \%)$. In order to reduce the hot-band contributions, the gas jet was cooled by flowing cold methanol through a copper cooling block that surrounded the gas jet. A $70 \mu \mathrm{m}$ diameter gas jet was used for these experiments, and the stagnation pressure was adjusted to maintain a chamber pressure of $2 \times 10^{-4}$ Torr. Higher pressures were found to introduce artifacts in the data owing to collisional effects. The fluorescence was dispersed by a SPEX optical monochromator (model $500 \mathrm{M}$ ) equipped with a $3600 \mathrm{~g} / \mathrm{mm}$ grating and detected by a liquid nitrogen-cooled CCD optical multichannel analyzer (Princeton Instruments LN/CCD-1340×400EBI). The detector is $25 \mathrm{~mm}$ wide, and the reciprocal linear dis-
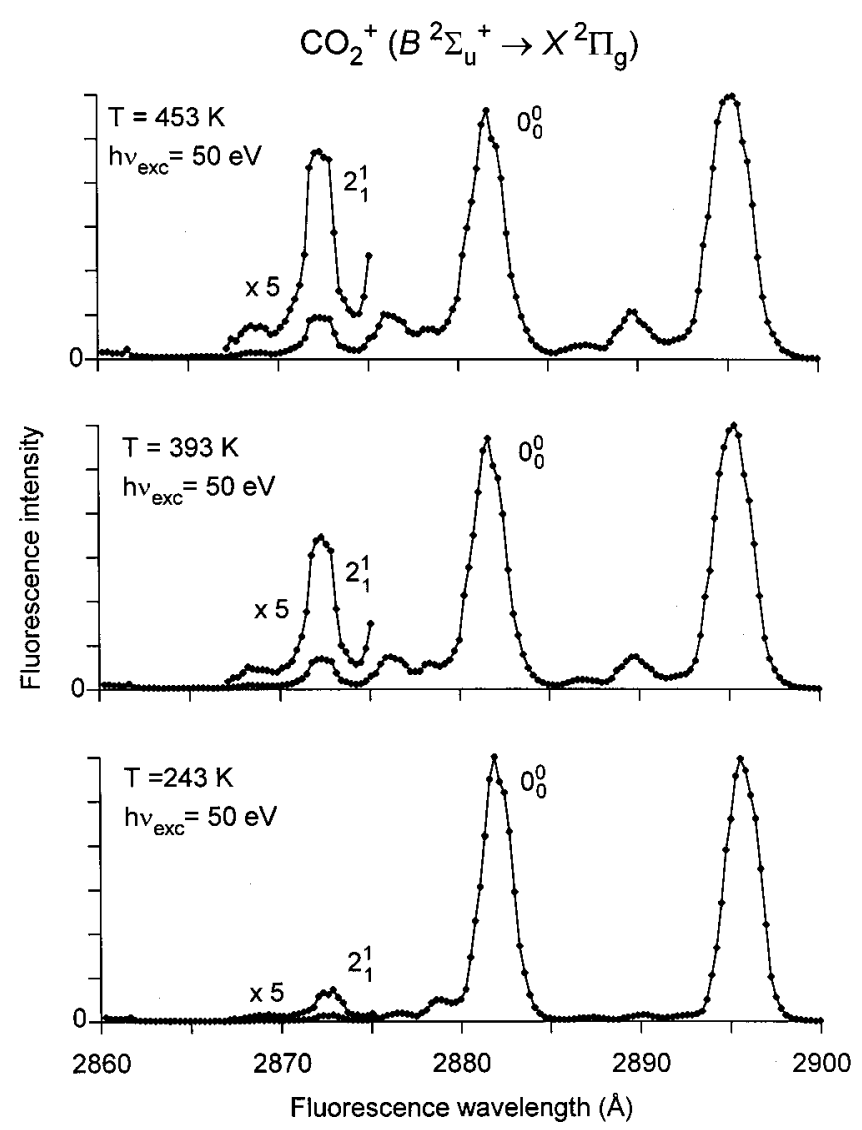

FIG. 2. Fluorescence spectrum following $\mathrm{CO}_{2} 3 \sigma_{u}^{-1}$ photoionization, with pertinent transitions labeled. The $2{ }_{1}^{1}$ and the $0_{0}^{0}$ transitions originate from the excited bending level $\left[v^{+}=(0,1,0)\right]$ and the ground vibrational level $\left[v^{+}\right.$ $=(0,0,0)]$, respectively. The spectra are displayed at different stagnation temperatures of the nozzle. As the temperature is increased, the population of the $(0,1,0)$ level in the sample gas increases. Ionization of this level creates the ion in the $v^{+}=(0,1,0)$ level, so the intensity of the $2{ }_{1}^{1}$ ionic fluorescence transition grows relative to that of the $0_{0}^{0}$ transition.

persion of the monochromator at these wavelengths is 5.3 $\AA / \mathrm{mm}$, resulting in a fluorescence spectral range of $130 \AA$. This was sufficient to include transitions from both the $v^{+}$ $=(0,0,0)$ and the $v^{+}=(0,1,0)$ levels simultaneously. This is useful because the vibrational branching ratios obtained in this way are not prone to significant systematic errors, such as variations in sample pressure. The entrance slit width of the fluorescence monochromator was set at $550 \mu \mathrm{m}$, corresponding to a bandwidth of $2.9 \AA$.

\section{EXPERIMENTAL RESULTS}

A portion of the $\mathrm{CO}_{2}^{+}\left(B^{2} \Sigma_{u}^{+} \rightarrow X^{2} \Pi_{g}\right)$ fluorescence spectrum is shown in Fig. 2. This fluorescence spectrum has been studied extensively. ${ }^{30,31}$ The fluorescence spectrum has two large peaks and they are both due to fluorescence from $v^{+}=(0,0,0)$. The ground state of $\mathrm{CO}_{2}^{+}$is an $X^{2} \Pi_{g}$ state, so spin-orbit splitting produces two terminal levels for the fluorescence transition. The results are the same regardless of which peak is used in the analysis. ${ }^{27}$ The data presented here employ the lower-wavelength component. The $v^{+}=(0,1,0)$ level fluoresces to the $v^{\prime \prime}=(0,1,0)$ level, and this level, $v^{\prime \prime}$ $=(0,1,0)$, is split into four components by Renner-Teller 


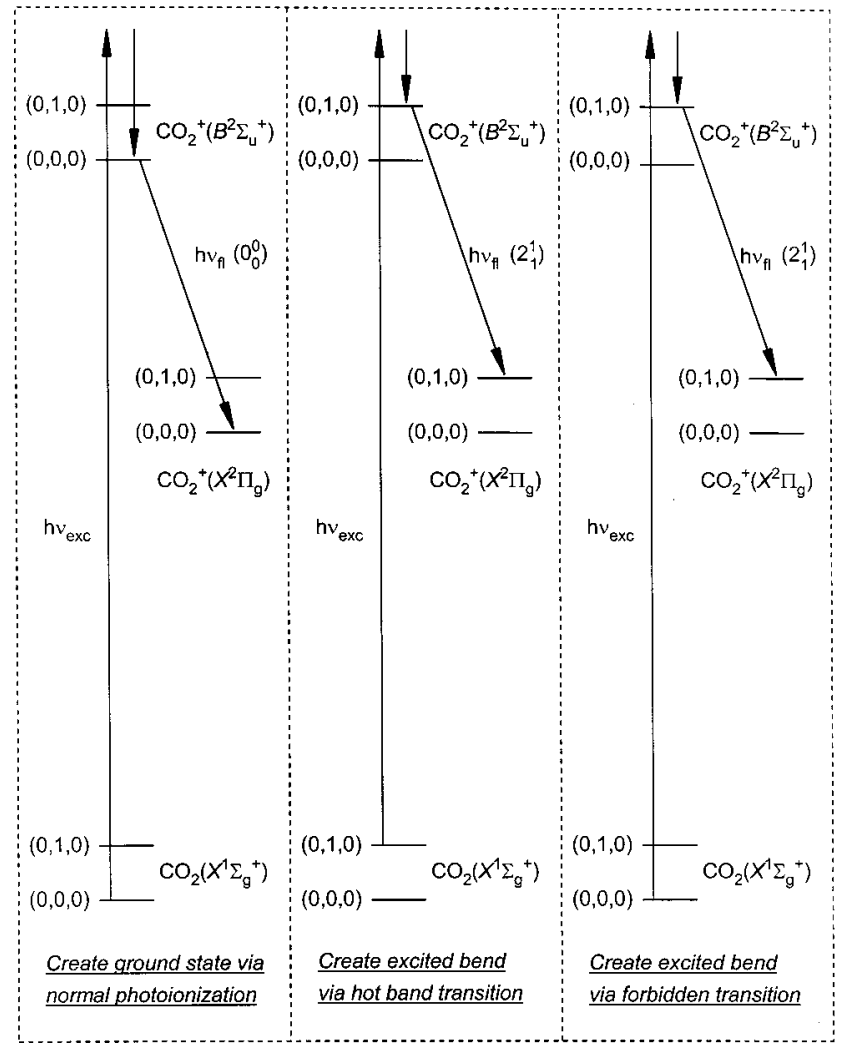

FIG. 3. Cartoon view illustrating the pertinent excitation/fluorescence pathways employed in this study.

coupling. The results are strongly temperature dependent, as seen in the spectra generated at different stagnation temperatures in Fig. 2. The $2{ }_{1}^{1}$ fluorescence transition is growing significantly as the temperature is increased, indicating that hot band ionization [i.e., photoionization of the $v_{0}=(0,1,0)$ level] is increasing. The three processes that need to be considered are sketched in Fig. 3. The dominant process is creation of the $B^{2} \Sigma_{u}^{+}, v^{+}=(0,0,0)$ level by ionization of ground vibrational state target molecules (left frame). The two pathways that create the $B^{2} \Sigma_{u}^{+}, v^{+}=(0,1,0)$ level resulting in the $2{ }_{1}^{1}$ fluorescence transition are the nominally forbidden photoionization transition (right frame) and hotband photoionization (middle frame).

A useful way to illustrate the relative importance of the alternate routes creating the $v^{+}=(0,1,0)$ level is to study the energy dependence of the intensity ratio as a function of stagnation temperature. This is done in Fig. 4 for the energy range $20 \leqslant h \nu_{\text {exc }} \leqslant 100 \mathrm{eV}$. These intensity ratio curves are scaled to one another for the purpose of comparison. The principal result from Fig. 4 is that there is a significant contribution from the nominally forbidden pathway responsible for creating the $\mathrm{CO}_{2}^{+}\left[B^{2} \Sigma_{u}^{+}, v^{+}=(0,1,0)\right]$ at the lower stagnation temperatures. If only the hot-band mechanism were operative, all of these curves would parallel each other. The fact that they display significant differences as the temperature is lowered demonstrates that the nominally forbidden pathway can be probed. However, the low-temperature limit has not been reached at $243 \mathrm{~K}$, the lowest temperature for which data were acquired. The $243 \mathrm{~K}$ data still have a contribution from hot-band ionization.
$\mathrm{CO}_{2}^{+}\left(B^{2} \Sigma_{\mathrm{u}}^{+}\right) \mathrm{v}^{+}=(0,1,0) / \mathrm{v}^{+}=(0,0,0)$ temperature dependence

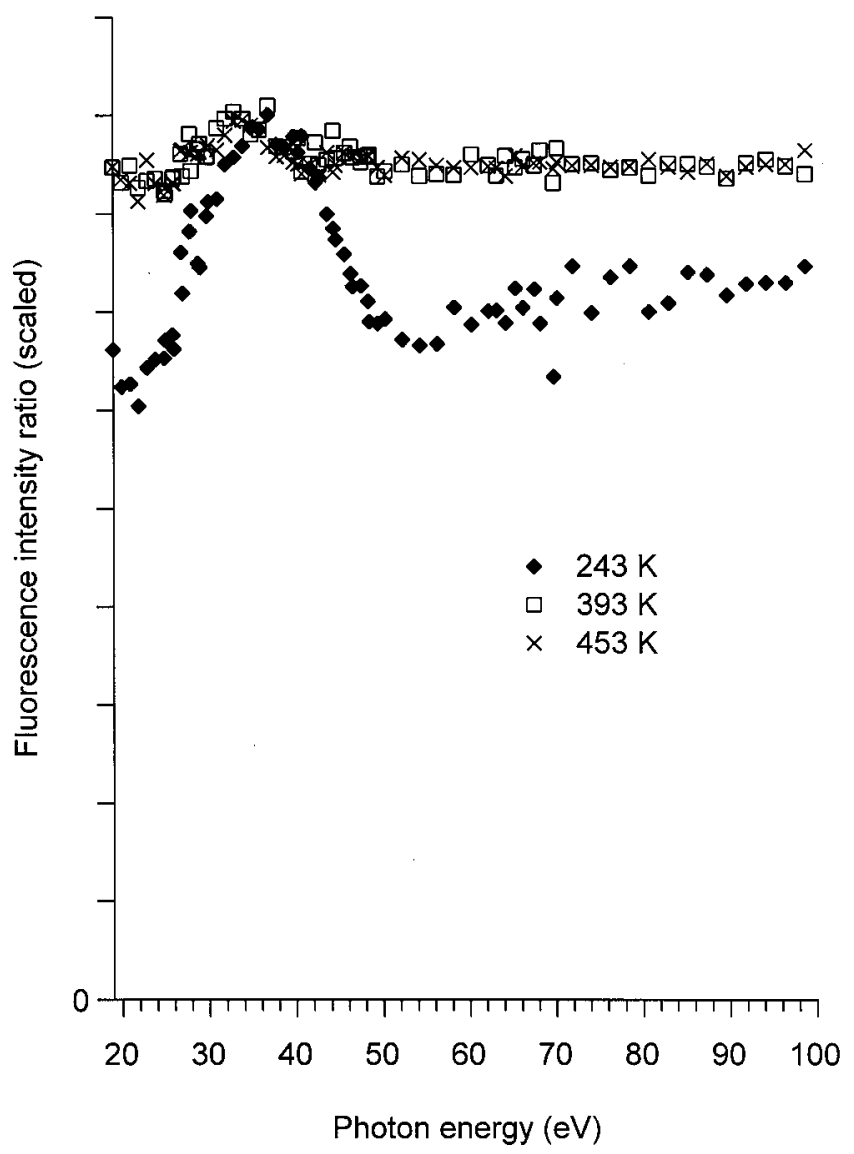

FIG. 4. Branching ratio for bending excitation following $\mathrm{CO}_{2} 3 \sigma_{u}^{-1}$ photoionization. These results are obtained by taking the $2{ }_{1}^{1} / 0_{0}^{0}$ fluorescence intensity ratio (see Figs. 2 and 3) as a function of energy. Results are shown at several stagnation temperatures to illustrate the importance of the vibrationally forbidden transitions at the lower temperatures.

Inspection of the lowest temperature result $(243 \mathrm{~K})$ in Fig. 4 shows that the ratio is still increasing at the highest energy $\left(h \nu_{\text {exc }}=100 \mathrm{eV}\right)$. As a result, we acquired data to higher energies at $243 \mathrm{~K}$, and the $v^{+}=(0,1,0) /(0,0,0)$ acquired over the photon energy range $18 \leqslant h \nu \leqslant 190 \mathrm{eV}$ is presented in Fig. 5. This is a primary result of this investigation, and the implications are described in the following sections. For now, we simply catalog some of the observations. First, the strong deviations in the branching ratio persist over the whole range. There is a pronounced dip at $h \nu_{\mathrm{exc}} \approx 21 \mathrm{eV}$. Additionally, a strong maximum appears near $h \nu_{\text {exc }}$ $\approx 36 \mathrm{eV}$. Finally, one of the most intriguing results is the very broad rise in the branching ratio extending from $80 \mathrm{eV}$ $\leqslant h \nu_{\text {exc }} \leqslant 200 \mathrm{eV}$. Theoretical results are also given in Fig. 5, but we defer the discussion of the comparison between experiment and theory until the theoretical approach is covered in the following section.

As mentioned previously, the (left) $y$ axis for the experimental data in Fig. 5 refers to the fluorescence intensity ratio for the two features labeled in Fig. 2. Even if there were no hot-band contributions to the production of the $v^{+}=(0,1,0)$ level, this ratio would $n o t$ be equal to the $(0,1,0) /(0,0,0)$ branching ratio. Fluorescence transitions from alternative vi- 


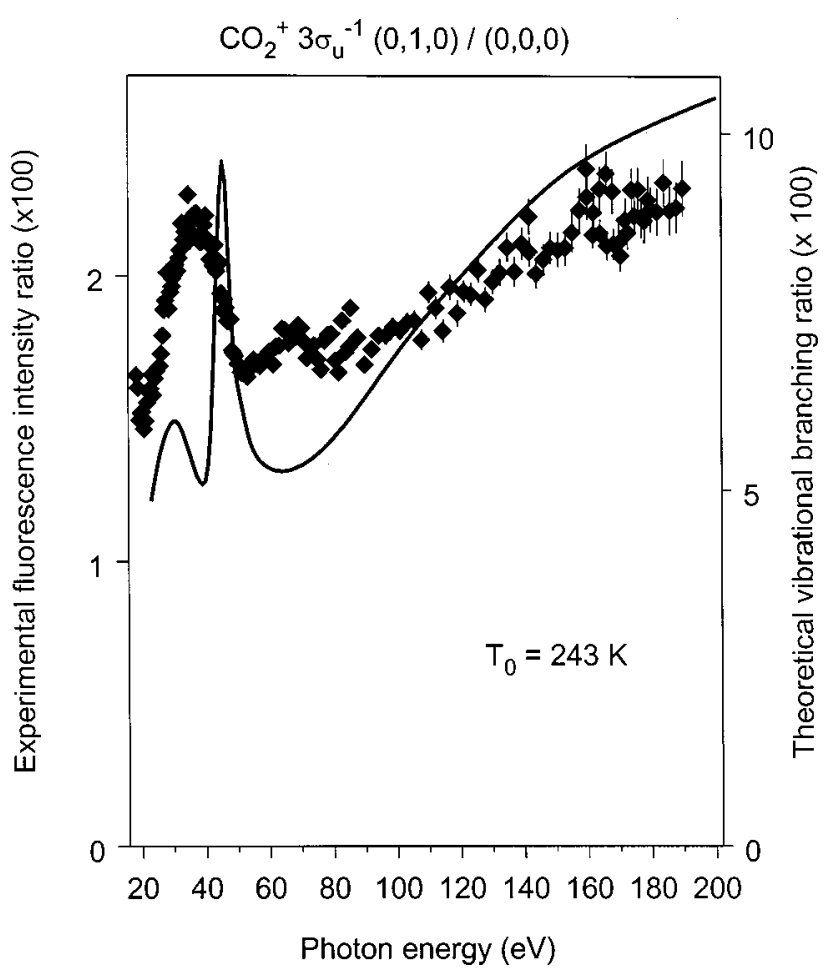

FIG. 5. Branching ratio for bending excitation following $\mathrm{CO}_{2} \quad 3 \sigma_{u}^{-1}$ photoionization. These results are obtained by taking the $2{ }_{1}^{1} / 0_{0}^{0}$ fluorescence intensity ratio (see Fig. 2) as a function of energy at the lowest stagnation temperature used in this investigation $(243 \mathrm{~K})$. The theory curve is discussed in the text.

brational levels will generally have different intensities, depending on a number of factors (e.g., Franck-Condon factors connecting upper and lower levels, different predissociation rates, etc.). ${ }^{27,32}$

\section{THEORY}

Previously, ${ }^{1}$ we found that vibronic coupling involving intensity borrowing from alternative ionic hole states was not sufficient to explain the energy dependence of the vibrational intensity ratio observed in Fig. 5. Specifically, it was shown that the energy-dependent vibrational branching ratio should track the $X^{2} \Pi_{g} / B^{2} \Sigma_{u}^{+}$electronic branching ratio ${ }^{33}$ if the usual interchannel coupling picture was assumed. In fact, the data did not support this approach. Thus, we attempt to clarify the origin of the forbidden transition with an alternative approach. According to Herzberg, ${ }^{34}$ an electronic transition is forbidden if the fixed-nuclei dipole matrix element (with the molecule in its equilibrium structure) connecting the initial and final states is zero. If the inclusion of the vibrational part of the Born-Oppenheimer molecular wave function in the dipole matrix element leads to a nonzero dipole matrix element, then this is referred to as a "type (a)" vibronic interaction and the transition can be observed. In the context of photoionization, such a type (a) vibronic interaction is a type of non-Franck-Condon effect involving molecular vibrations that lower the symmetry of the molecule. Thus, in the photoionization of $\mathrm{CO}_{2}$, it is possible to observe forbidden vibronic photoionization processes involving the excitation of the bending or antisymmetric stretching modes.
To our knowledge, the effects of a "type (a)" vibronic interaction have never been quantified for a photoionization transition i.e., with explicit consideration of the effects of the continuum electron. As a result, such an effect has never been identified unambiguously as a production mechanism for a nominally forbidden vibrational transition in photoionization. The possibility of such an intrachannel mechanism was suggested by Stimson et al., ${ }^{11}$ where they noted that a Franck-Condon breakdown might be responsible for the observation of singly excited bending levels in ZEKE-PFI spectra of OCS. The importance of this picture-i.e., Franck-Condon breakdown as suggested previously ${ }^{11}$ and quantified here-is that the "type (a)" mechanism is an intrachannel phenomenon, i.e., no oscillator strength is transferred between alternative electronic channels. The key point is that the computation is performed within the BornOppenheimer framework, which is conceptually and computationally straightforward. In the next section, we use this approach to compute the cross section for the forbidden photoionization of the $v_{0}=(0,0,0) X^{1} \Sigma_{g}^{+}$state of $\mathrm{CO}_{2}$, leading to the $v^{+}=(0,1,0) B^{2} \Sigma_{u}^{+}$state of $\mathrm{CO}_{2}^{+}$. These results will be combined with the computed cross sections for the corresponding $v_{0}=(0,0,0) \rightarrow v^{+}=(0,0,0)$ and $v_{0}=(0,1,0)$ $\rightarrow v^{+}=(0,1,0)$ photoionization processes to yield temperature-dependent emission branching rations that can then be compared to the experimental data.

\section{A. Explicit form of the vibronic matrix elements}

To consider the effects of the type (a) vibronic coupling in the photoionization of $\mathrm{CO}_{2}$, we will only treat the degenerate bending motion and neglect the stretching motions. In Fig. 6 we give the coordinate definitions for the various angles that we need to consider. We have drawn the angles of the molecule and the photoelectron relative to a fixed frame of reference in which the axis perpendicular to the figure is the $a$ axis, which is the principal axis of the molecule that coincides with the molecular axis when the molecule is linear. The angle $\phi_{k}^{\prime}$ is the angle between the vector given by the intersection of the plane of the molecule and the plane perpendicular to the $a$ axis given in Fig. 6 and the vector that is the projection of the direction of the photoelectron into the plane perpendicular to the $a$ axis. The angle $\phi_{k}$ is the corresponding angle between the projected direction of the photoelectron and the fixed molecular frame axis. We will use the corresponding definitions for $\phi_{n}^{\prime}$ and $\phi_{n}$ to define the orientation of the polarization of the light. The angle $\gamma$ is the angle between the molecular plane and the fixed molecular frame axis. The angle $\gamma$ thus describes vibrational angular motion that will be characterized by the vibrational angular momentum quantum numbers $l_{i}$ and $l_{f}$ in the initial and final states, respectively. The last coordinate is the coordinate $x$ that will be used to indicate the displacement of the $\mathrm{C}$ atom from the $a$ axis.

All calculated photoionization amplitudes are computed with the molecule oriented such that $\gamma=0$. In this orientation, the amplitude for photoionization has the partial-wave expansion of the form 


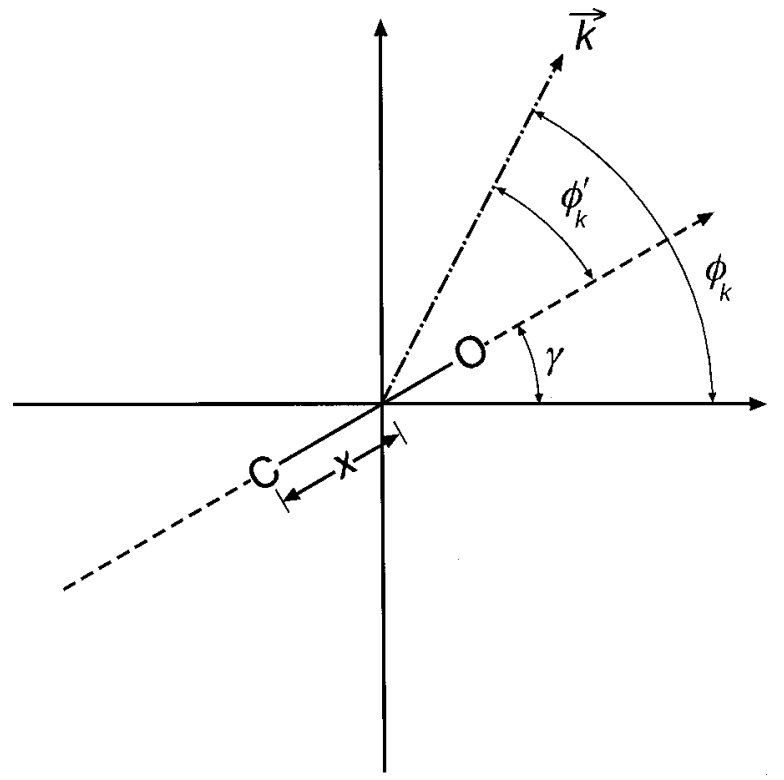

FIG. 6. Coordinate system used in the derivation of the vibronic matrix elements. The axes given here define a fixed molecular frame plane that is perpendicular to the molecular $a$ axis. The vector $k$ is the projection of the photoelectron direction in the plane of the figure. The molecule is drawn as its projection in the plane. The distance $x$ is the displacement of the $\mathrm{C}$ atom from the $a$ axis. The angle $\gamma$ is the angle between the plane of the molecule and the fixed molecular frame axis and thus describes the vibrational angular motion. The angle $\phi_{k}$ is then the angle between the projection of the direction of the photoelectron in the plane and the fixed molecular axis. The angle $\phi_{k}^{\prime}$ is the corresponding angle between the projection of the direction of the photoelectron in the plane of the figure and the plane containing the molecule.

$$
f(\hat{k}, \hat{n}, x, \gamma=0)=\sum_{l, m, \mu} I_{l m \mu}(x, \gamma=0) Y_{l m}^{*}(\hat{k}) Y_{1 \mu}^{*}(\hat{n}),
$$

where $\hat{k}$ is the direction of emission of the photoelectron and $\hat{n}$ is the direction of the polarization of the light. The amplitude for photoionization with an arbitrary value of $\gamma$ can then be obtained by rotation about the $a$ axis to give

$f(\hat{k}, \hat{n}, x, \gamma)$

$$
=\sum_{l, m, \mu} I_{l m \mu}(x, \gamma) Y_{l m}^{*}\left(\theta_{\hat{k}}, \phi_{\hat{k}}-\gamma\right) Y_{1 \mu}^{*}\left(\theta_{\hat{n}}, \phi_{\hat{n}}-\gamma\right),
$$

where we have assumed that the electronic initial and ion states have $\Sigma$ symmetry. Using the properties of the spherical harmonics, this can be written as

$f(\hat{k}, \hat{n}, x, \gamma=0)=\sum_{l, m, \mu} I_{l m \mu}(x, \gamma=0) e^{i(m+\mu) \gamma} Y_{l m}^{*}(\hat{k}) Y_{1 \mu}^{*}(\hat{n})$.

If the initial and final bending states are written as $\chi_{v_{i}, l_{i}}^{(i)}(x) e^{i l_{i} \gamma}$ and $\chi_{v_{f}, l_{f}}^{(f)}(x) e^{i l_{f} \gamma}$, then the vibrationally averaged amplitude is given by

$f^{(f \leftarrow i)}(\hat{k}, \hat{n})=\left\langle\chi_{v_{i}, l_{i}}^{(i)}(x) e^{i l_{i} \gamma}|f(\hat{k}, \hat{n}, x, \gamma)| \chi_{v_{f}, l_{f}}^{(f)}(x) e^{i l_{f} \gamma}\right\rangle_{x, \gamma}$.

If the vibrationally averaged amplitude is partial-wave expanded in the form

$$
f^{(f \leftarrow i)}(\hat{k}, \hat{n})=\sum_{l, m, \mu} I_{l, m, \mu}^{(f \leftarrow i)} Y_{l m}^{*}(\hat{k}) Y_{1 \mu}^{*}(\hat{n}),
$$

the vibrationally averaged partial-wave amplitudes are then given by

$$
\begin{aligned}
& f_{l m \mu}^{(f \leftarrow i)} \\
& \quad=\left\langle\chi_{v_{i}, l_{i}}^{(i)}(x) e^{i l_{i} \gamma}\left|I_{l m \mu}(x, \gamma=0) e^{i(m+\mu) \gamma}\right| \chi_{v_{f}, l_{f}}^{(f)}(x) e^{i l_{f} \gamma}\right\rangle_{x, \gamma} .
\end{aligned}
$$

Equation (7) can then be integrated over $\gamma$ to give

$l_{l m \mu}^{(f \leftarrow i)}=\left\langle\chi_{v_{i}, l_{i}}^{(i)}(x)\left|I_{l m \mu}(x, \gamma=0)\right| \chi_{v_{f}, l_{f}}^{(f)}(x)\right\rangle_{x} \delta_{l_{i}, l_{f}+m+\mu}$.

The matrix elements given in Eq. (8) are then used to compute the orientationally averaged cross sections in the usual manner. ${ }^{35}$

Finally, we comment on how the nominally forbidden transitions to the $v^{+}=(0,1,0)$ level become allowed, and even enhanced. In Eq. (8) the matrix elements that give the intensity of allowed vibronic transitions have $m+\mu=0$. In the equilibrium linear geometry for ionization from the $3 \sigma_{u}$ orbital in $\mathrm{CO}_{2}$, the only nonzero values of $I_{l m \mu}$ have even values of $l$ and $m+\mu=0$, thus only they lead to the allowed transitions. Intensity leading to the forbidden vibronic transition $v_{0}=(0,0,0) \rightarrow v^{+}=(0,1,0)$ is obtained from matrix elements with $l_{i}=0$ and $l_{f}=1$. Thus, for the forbidden transition we have $m+\mu=-1$. As the molecule bends, one finds nonzero values of $I_{l m \mu}$ with $m+\mu=-1$ and with odd values of $l$. Which combinations of $l, m$ and $\mu$ lead to nonzero matrix elements is controlled by the symmetry of the bent molecule, $C_{2 v}$ in the case of $\mathrm{CO}_{2}$. Note that this formulation will lead to nonzero values of $I_{\operatorname{lm} \mu}$ if and only if we do not factor the electronic part from the vibrational part, i.e., if we do not invoke the Franck-Condon approximation following Eq. (8). As will be discussed later, the matrix elements leading to the forbidden transitions can be enhanced by the presence of a resonance in the scattering continuum, thus leading to the resonance enhanced forbidden transitions.

\section{B. Calculated results}

The target wave function for $\mathrm{CO}_{2}$ was a Hartree-Fock wave function computed using the Gaussian $\operatorname{code}^{36}$ with an aug-cc-pVTZ basis set. ${ }^{37,38}$ Four geometries were computed with the angle $\angle \mathrm{O}-\mathrm{C}-\mathrm{O}$ having the values $180^{\circ}, 175^{\circ}, 170^{\circ}$, and $165^{\circ}$ and $R(\mathrm{C}-\mathrm{O})=1.1612 \AA{ }^{34}$ The corresponding fixed-nuclei photoionization cross sections for ionization leading to the $3 \sigma_{u}^{-1} B^{2} \Sigma_{u}^{+}$state of $\mathrm{CO}_{2}^{+}$were computed using the Schwinger variational method with all matrix elements evaluated with the single-center expansion method. All calculations were performed using the single-channel frozen-core Hartree-Fock approximation (SCFCHF). This method has been discussed in detail previously and will not be discussed further here. ${ }^{35,39,40}$ In all matrix elements, the wave functions were expanded up to a partial wave of $l_{\max }$ $=20$ and all terms were retained in the expansions of the static and exchange integrals consistent with this value of $l_{\max }$. All calculations were done with the $\mathrm{C}$ atom as the 


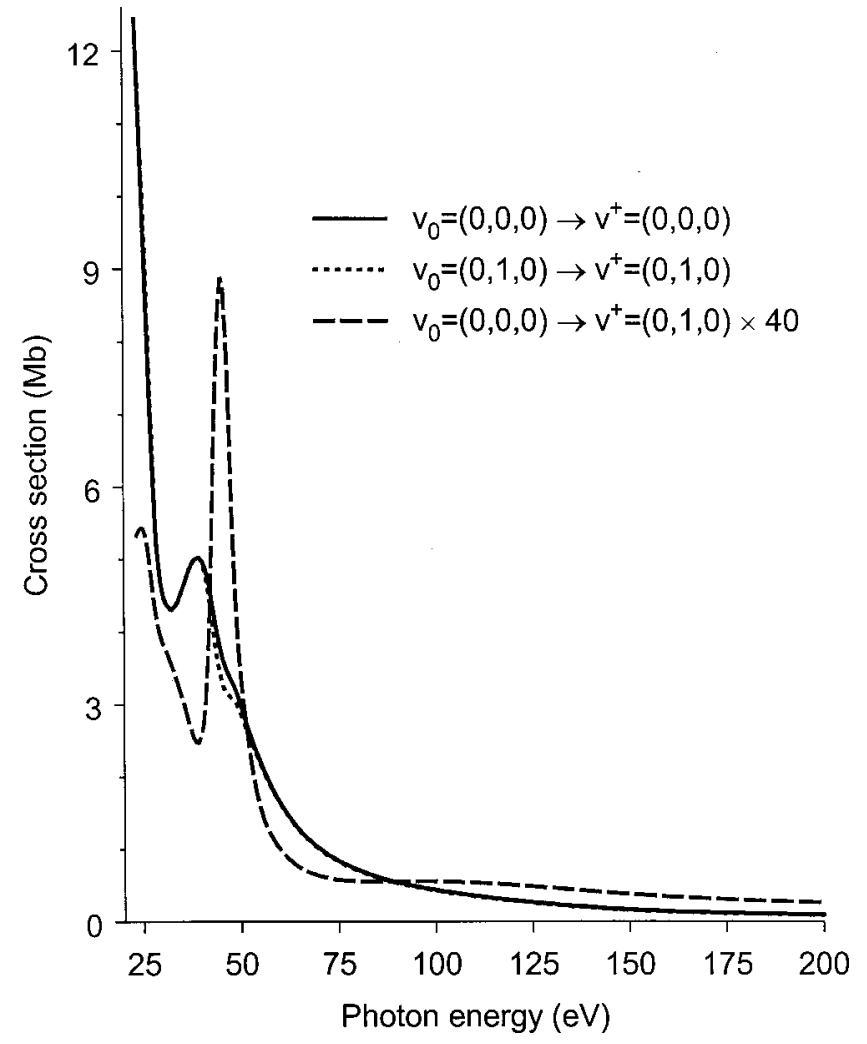

FIG. 7. Cross sections for the $v_{0}=(0,0,0) \rightarrow v^{+}=(0,0,0), v_{0}=(0,0,0)$ $\rightarrow v^{+}=(0,1,0)$, and $v_{0}=(0,1,0) \rightarrow v^{+}=(0,1,0)$ photoionization of $\mathrm{CO}_{2}$ leading to the $3 \sigma_{u}^{-1} B^{2} \Sigma_{u}^{+}$state of $\mathrm{CO}_{2}^{+}$.

center of expansion. The resulting dipole matrix should be invariant to the translation of mass of the molecule. Thus, they should be the same with the center of the single-center expansion at the $\mathrm{C}$ atom or at the center of mass of the molecule. This is not strictly true in our calculations due to the numerical approximations inherent in the single-center expansion. The bending vibrational wave functions were taken to be the two-dimensional harmonic oscillator functions using a harmonic frequency of $667.4 \mathrm{~cm}^{-1} .34$

In Fig. 7 we give the cross sections for the $v_{0}=(0,0,0)$ $\rightarrow v^{+}=(0,0,0), \quad v_{0}=(0,0,0) \rightarrow v^{+}=(0,1,0), \quad$ and $\quad v_{0}$ $=(0,1,0) \rightarrow v^{+}=(0,1,0)$ photoionization of $\mathrm{CO}_{2}$ leading to the $3 \sigma_{u}^{-1} B^{2} \Sigma_{u}^{+}$state of $\mathrm{CO}_{2}^{+}$. Previous calculations using the SCFCHF approximation have found ${ }^{2}$ a narrow resonance that occurs in the $\sigma_{u}$ continuum at a photoelectron kinetic energy of about $24 \mathrm{eV}$. This feature is not observable in the $3 \sigma_{u}^{-1}$ photoionization of $\mathrm{CO}_{2}$ without invoking bending, owing to violation of the $g \leftrightarrow u$ selection rule, but it is well known for $4 \sigma_{g}^{-1}$ photoionization of $\mathrm{CO}_{2}$. Additional studies have shown that averaging over the vibrational state ${ }^{3,41}$ and including interchannel coupling ${ }^{4}$ increases the width of this feature in the cross section. Thus, in the ionization leading to the $4 \sigma_{g}^{-1} C^{2} \Sigma_{g}^{+}$state of $\mathrm{CO}_{2}^{+}$with an ionization energy (IE) of $18.2 \mathrm{eV}$, there is a prominent shape resonance at a photon energy of $42 \mathrm{eV}$. In the photoionization of $\mathrm{CO}_{2}$ computed using the fixed-nuclei approximation in the linear geometry, there is a broad feature in the ionization leading to the $3 \sigma_{u}^{-1} B^{2} \Sigma_{u}^{+}$state of $\mathrm{CO}_{2}^{+}$at around a photon energy of 40 $\mathrm{eV}$. This feature is evident in the $v_{0}=(0,0,0) \rightarrow v^{+}$

\section{$\mathrm{CO}_{2} 3 \sigma_{\mathrm{u}}^{-1}$ relative production rates at different target temperatures}

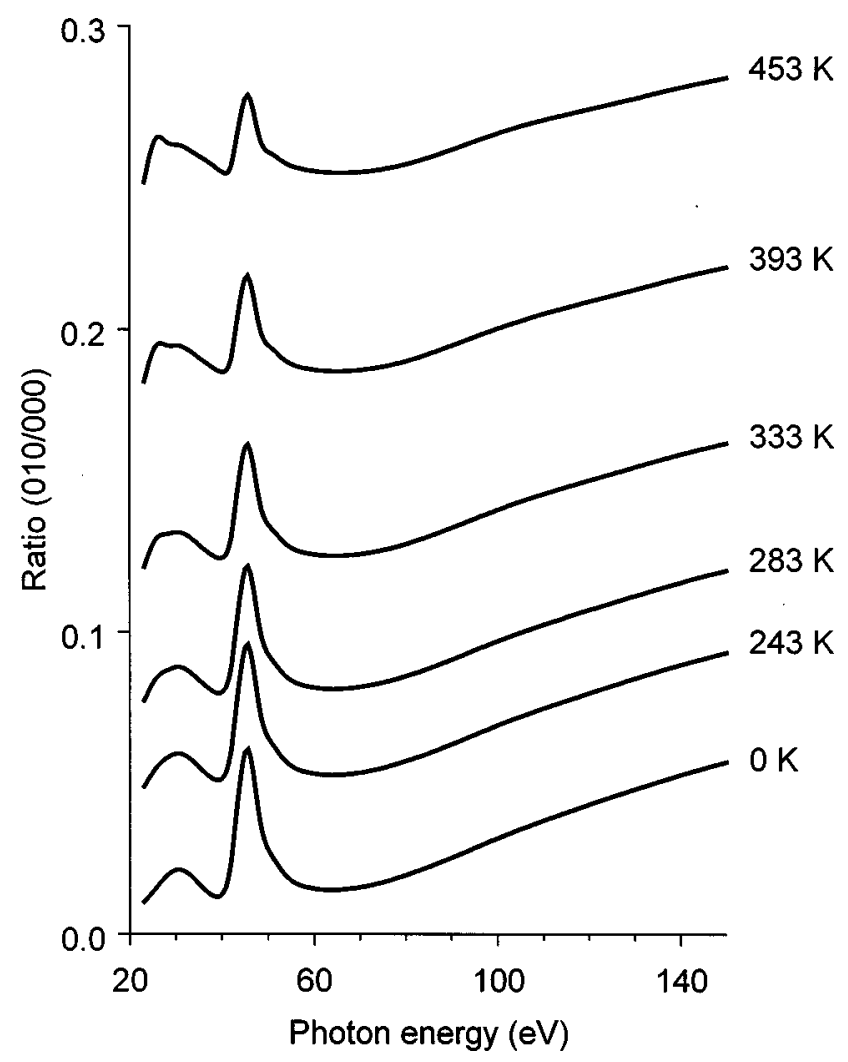

FIG. 8. Theoretical ratio of the population of $v^{+}=(0,1,0)$ to $v^{+}=(0,0,0)$ in the $3 \sigma_{u}^{-1} B^{2} \Sigma_{u}^{+}$state of $\mathrm{CO}_{2}^{+}$as a function of the initial vibrational temperature.

$=(0,0,0)$ and $v_{0}=(0,1,0) \rightarrow v^{+}=(0,1,0)$ cross sections shown in Fig. 7. When the molecule is bent, the $3 \sigma_{u}$ orbital acquires a small amount of $\pi_{g}$ character and the resonant $\sigma_{u}$ state acquires a small amount of $\pi_{g}$ character, thus allowing there to be intensity leading the $\sigma_{u}$ resonant state. This is the source of the narrow feature seen in the computed $v_{0}$ $=(0,0,0) \rightarrow v^{+}=(0,1,0)$ cross section shown in Fig. 7.

In Fig. 8 we give the predicted temperature-dependent ratio of the population of $v^{+}=(0,1,0)$ to $v^{+}=(0,0,0)$ in the $3 \sigma_{u}^{-1} B^{2} \Sigma_{u}^{+}$state of $\mathrm{CO}_{2}^{+}$, assuming that only the three cross sections shown in Fig. 7 contribute and assuming that the population of the initial vibrational state is given by a thermal distribution at the indicated temperature. We can see that the peak to background ratio of the feature at $46 \mathrm{eV}$ dramatically decreases as the temperature increases, as seen in the experiment. Finally, returning to Fig. 5, we compare the $T$ $=243 \mathrm{~K}$ prediction to the unnormalized experimental data obtained with a stagnation temperature of $243 \mathrm{~K}$. We can see that there is fair agreement between experiment and theory in this figure. The calculations are fairly simple and thus there are a number of possible sources for the discrepancy between theory and experiment. There are correlation effects such as interchannel coupling and initial and final state correlation. There are also neglected effects due to the symmetric and antisymmetric stretching modes. Both of these effects are 
known to broaden the width of the predicted feature, and thus should improve the agreement between experiment and theory.

\section{DISCUSSION}

\section{A. Overview}

The results shown in Fig. 5 are encouraging, and it is useful to summarize some of the lessons that can be extracted from these data. First, we see that calculations at the independent particle level of approximation (i.e., HartreeFock in the continuum) is sufficient to reproduce the major trends in the experimental data. This is a novel and surprising result, as the excitation of the bending vibration with a single quantum is a nominally forbidden transition and interchannel effects are usually invoked to explain such observations. This mechanism, photoelectron-induced vibronic symmetry breaking, appears to be generally applicable, and might even be extended to analogous (nominally) forbidden ionization transitions in nonlinear systems. We emphasize that generating the energy-dependent data is extremely useful in guiding us to the underlying physics of the process. Second, the "low" energy results show sensitivity of shape resonance to bond angle. While it is well established that shape resonances are affected by changes in bond length, there has been little, if any, attention paid to possible correlations between shape resonant ionization dynamics and other types of mode-specific nuclear motions. ${ }^{41}$ Finally, the dependence of the vibrational ratio at high energies is very curious. Indeed, the ratio is still rising even at photon energies above $190 \mathrm{eV}$ (i.e., more than $170 \mathrm{eV}$ above the threshold). It is not clear if the vibrational ratio has leveled out at these highest energies, or if it is still rising. While the broad features at low energy are almost certainly due to shape resonant channels, the higher-energy behavior is not yet assigned, and it requires further scrutiny to interpret. In a previous study on photoionization for $\mathrm{N}_{2}$, we found that the vibrational branching ratios varied over a similarly expansive range, owing to the sensitivity of Cooper minima to internuclear separation. ${ }^{26}$ It is possible that a related phenomenon is manifested in the $\mathrm{CO}_{2}$ case.

While the agreement between experiment and theory is good, a few words of caution are in order. First, it could be that more than a single mechanism is responsible for creation of the $v^{+}=(0,1,0)$ vibrational level, including interchannel coupling effects. Indeed, in the previous work of Roy et al. on $4 \sigma_{g}^{-1}$ photoionization of $\mathrm{CO}_{2},{ }^{14}$ their interpretation of interchannel coupling was successful in explaining the energy dependence of the photoelectron asymmetry parameter for the $v^{+}=(1,0,1)$ level. An implication is that interchannel effects could be playing a role in the current study, as well. Second, the agreement between experiment and theory is rather good, but there are disagreements that require further scrutiny. In particular, the enhancement in the vibrational ratio occurs at higher energy in the theoretical treatment. This could indicate that the theory is overestimating the contribution of the symmetry-breaking $k \sigma_{u}$ continuum channel, and underestimating the effect of bond angle on the $k \sigma_{g}$ channel. Similarly, on the experimental side, the study would benefit from lower vibrational temperatures to reduce the hot-band transition even further. While these lowertemperature studies are desirable, it is important to note that the main features are reproduced well in Fig. 5 with the current experimental results.

\section{B. Connections to related spectroscopies}

Photoionization dynamics play a central role in VUV and soft $\mathrm{x}$-ray spectroscopy. In particular, an understanding of shape resonant dynamics for valence shell processes has not only developed fundamental insights into scattering theory, but also helped to clarify applications of XANES spectroscopy. ${ }^{23}$ Indeed, the early theoretical ${ }^{20}$ and experimental work $^{21}$ into Franck-Condon breakdown following valence-shell shape resonant excitation has been exploited as a tool to develop XANES for probing geometry changes of adsorbates on solid surfaces. ${ }^{22-25}$ For polyatomic systems, however, there is comparatively little work on vibrationally state-resolved valence shell photoionization, and the current work provides qualitatively new material to such studies. Research on polyatomic systems have been shown to address novel issues, as experiment ${ }^{32,42}$ and theory ${ }^{43,44}$ demonstrated that vibrationally mode-specific effects were exhibited in $\mathrm{N}_{2} \mathrm{O} 7 \sigma^{-1}$ photoionization. This idea was similarly applied to $\mathrm{CS}_{2}{ }^{45}$ Mode-specific investigations of polyatomic systems are extremely useful because one can test the response of the ionization dynamics to specific vibrational motions. There have been only a few polyatomic systems that have been characterized at energies more than $10 \mathrm{eV}$ above threshold with vibrational resolution: $\mathrm{N}_{2} \mathrm{O},{ }^{32,42-44,46} \mathrm{SiF}_{4},{ }^{47}$ $\mathrm{CS}_{2},{ }^{48} \mathrm{CF}_{4},{ }^{49} \mathrm{C}_{2}(\mathrm{CN})_{2},{ }^{50}$ and $\mathrm{C}_{6} \mathrm{~F}_{6},{ }^{51}$ and most of these studies have focused on stretching (or analogously, breathing) motions. In the current study we extend those earlier results in a significant way by showing how previously neglected vibrational modes can be exploited for such mode-specific investigations. Vibrationally state-resolved studies elucidate connections between ionization dynamics and bond angle changes, and this can be useful in extending our understanding of core-shell spectroscopies, including XANES and EXAFS.

\section{CONCLUSIONS}

We report studies on the deposition of vibrational energy for a forbidden vibrational mode following $3 \sigma_{u}^{-1}$ photoionization of $\mathrm{CO}_{2}$. This was accomplished using vibrationally resolved dispersed fluorescence detection following photoionization, and the results demonstrate that the excited bending vibration is excited with an energy dependence that is surprising. In particular, theory and calculations indicate that the major energy-dependent features and trends can be explained rather well using a framework at the Hartree-Fock level of detail, i.e., no interchannel interactions are included. This explanation, photoelectron-induced vibronic symmetry breaking, has not been invoked previously to explain the existence of nominally forbidden transitions in photoelectron spectra, and it appears that such effects might be comparatively common. Refinements to the theory and the experiment are possible, and both are underway. 


\section{ACKNOWLEDGMENTS}

We thank the CAMD staff for their assistance, and EDP acknowledges support from the National Science Foundation (CHE-9616908). This work was also supported in part by the Robert A. Welch Foundation (Houston, Texas) under Grant No. A-1020. We also acknowledge the support of the Texas A\&M University Supercomputing Facility. Finally, we wish to acknowledge helpful conversations with Dr. M. G. White of Brookhaven National Laboratory.

${ }^{1}$ J. S. Miller and E. D. Poliakoff, J. Chem. Phys. 113, 899 (2000).

${ }^{2}$ R. R. Lucchese and V. McKoy, Phys. Rev. A 26, 1406 (1982).

${ }^{3}$ R. R. Lucchese and V. McKoy, Phys. Rev. A 26, 1992 (1982).

${ }^{4}$ R. R. Lucchese, J. Chem. Phys. 92, 4203 (1990).

${ }^{5}$ L. S. Wang, J. E. Reutt, Y. T. Lee, and D. A. Shirley, J. Electron Spectrosc. Relat. Phenom. 47, 167 (1988).

${ }^{6}$ H. C. Choi, R. M. Rao, A. G. Mihill, S. Kakar, E. D. Poliakoff, K. Wang, and V. McKoy, Phys. Rev. Lett. 72, 44 (1994).

${ }^{7}$ E. D. Poliakoff, H. C. Choi, R. M. Rao, A. G. Mihill, S. Kakar, K. Wang, and V. McKoy, J. Chem. Phys. 103, 1773 (1995).

${ }^{8}$ B. Wannberg, H. Veenhuizen, L. Mattsson, K. E. Norell, L. Karlsson, and K. Siegbahn, J. Phys. B 17, L259 (1984).

${ }^{9}$ F. Merkt, S. R. Mackenzie, R. J. Rednall, and T. P. Softley, J. Chem. Phys. 99, 8430 (1993).

${ }^{10}$ J. C. Huang, Y. S. Cheung, M. Evans, C. X. Liao, C. Y. Ng, C. W. Hsu, P. Heimann, H. Lefebvre-Brion, and C. Cossart-Magos, J. Chem. Phys. 106, 864 (1997).

${ }^{11}$ S. Stimson, M. Evans, C. Y. Ng, C. W. Hsu, P. Heimann, C. Destandau, G. Chambaud, and P. Rosmus, J. Chem. Phys. 108, 6205 (1998).

${ }^{12}$ J. B. Liu, W. W. Chen, C. W. Hsu, M. Hochlaf, M. Evans, S. Stimson, and C. Y. Ng, J. Chem. Phys. 112, 10767 (2000).

${ }^{13}$ J. B. Liu, M. Hochlaf, and C. Y. Ng, J. Chem. Phys. 113, 7988 (2000).

${ }^{14}$ P. Roy, R. J. Bartlett, W. J. Trela, T. A. Ferrett, A. C. Parr, S. H. Southworth, J. E. Hardis, V. Schmidt, and J. L. Dehmer, J. Chem. Phys. 94, 949 (1991).

${ }^{15}$ D. M. P. Holland, M. A. MacDonald, M. A. Hayes, P. Baltzer, L. Karlsson, M. Lundqvist, B. Wannberg, and W. von Niessen, Chem. Phys. 188, 317 (1994).

${ }^{16}$ P. Baltzer, L. Karlsson, M. Lundqvist, B. Wannberg, D. M. P. Holland, and M. A. Macdonald, Chem. Phys. 195, 403 (1995).

${ }^{17}$ D. M. P. Holland, M. A. Macdonald, P. Baltzer, L. Karlsson, M. Lundqvist, B. Wannberg, and W. von Niessen, Chem. Phys. 192, 333 (1995).

${ }^{18}$ D. M. P. Holland, M. A. MacDonald, M. A. Hayes, L. Karlsson, B. Wannberg, and P. Baltzer, J. Phys. B 29, 487 (1996).

${ }^{19}$ D. M. P. Holland, M. A. MacDonald, M. A. Hayes, L. Karlsson, and B. Wannberg, Chem. Phys. 226, 351 (1998).

${ }^{20}$ J. L. Dehmer, D. Dill, and S. Wallace, Phys. Rev. Lett. 43, 1005 (1979).

${ }^{21}$ J. B. West, A. C. Parr, B. E. Cole, D. L. Ederer, R. Stockbauer, and J. L. Dehmer, J. Phys. B 13, L105 (1980).
${ }^{22}$ J. Stöhr, F. Sette, and A. L. Johnson, Phys. Rev. Lett. 53, 1684 (1984).

${ }^{23}$ J. Stöhr, NEXAFS Spectroscopy (Springer-Verlag, Berlin, 1992).

${ }^{24}$ M. N. Piancastelli, D. W. Lindle, T. A. Ferrett, and D. A. Shirley, J. Chem. Phys. 86, 2765 (1987).

${ }^{25}$ M. N. Piancastelli, J. Electron Spectrosc. Relat. Phenom. 100, 167 (1999).

${ }^{26}$ R. M. Rao, E. D. Poliakoff, K. Wang, and V. McKoy, Phys. Rev. Lett. 76, 2666 (1996)

${ }^{27}$ E. D. Poliakoff, M.-H. Ho, G. E. Leroi, and M. G. White, J. Chem. Phys. 84, 4779 (1986).

${ }^{28}$ R. L. Stockbauer, P. Ajmera, E. D. Poliakoff, B. C. Craft, and V. Saile, Nucl. Instrum. Methods 291, 505 (1990).

${ }^{29}$ E. Morikawa, J. D. Scott, E. D. Poliakoff, R. L. Stockbauer, and V. Saile, Rev. Sci. Instrum. 63, 1300 (1992).

${ }^{30}$ M. A. Johnson, R. N. Zare, J. Rostas, and S. Leach, J. Chem. Phys. 80, 2407 (1984).

${ }^{31}$ M. A. Johnson, J. Rostas, and R. N. Zare, Chem. Phys. Lett. 92, 225 (1982).

${ }^{32}$ L. A. Kelly, L. M. Duffy, B. Space, E. D. Poliakoff, P. Roy, S. H. Southworth, and M. G. White, J. Chem. Phys. 90, 1544 (1989).

${ }^{33}$ M. R. F. Siggel, J. B. West, M. A. Hayes, A. C. Parr, J. L. Dehmer, and I. Iga, J. Chem. Phys. 99, 1556 (1993).

${ }^{34}$ G. Herzberg, Electronic Spectra and Electronic Structure of Polyatomic Molecules (Krieger, Malabar, 1991).

${ }^{35}$ A. P. P. Natalense and R. R. Lucchese, J. Chem. Phys. 111, 5344 (1999).

${ }^{36}$ M. J. e. a. Frisch, revision A6, Gaussian, Inc., Pittsburgh, 1998.

${ }^{37}$ T. H. Dunning, J. Chem. Phys. 90, 1007 (1989).

${ }^{38}$ R. A. Kendall, T. H. Dunning, and R. J. Harrison, J. Chem. Phys. 96, 6796 (1992).

${ }^{39}$ F. A. Gianturco, R. R. Lucchese, and N. Sanna, J. Chem. Phys. 100, 6464 (1994).

${ }^{40}$ F. A. Gianturco, R. R. Lucchese, N. Sanna, and A. Talamo, in Electron Collisions with Molecules, Clusters, and Surfaces, edited by H. Ehrhardt and L. A. Morgan (Plenum, New York, 1994), p. 71.

${ }^{41}$ C.-H. Yu, R. M. Pitzer, and C. W. McCurdy, J. Phys. Chem. 92, 3116 (1988).

${ }^{42}$ T. A. Ferrett, A. C. Parr, S. H. Southworth, J. E. Hardis, and J. L. Dehmer, J. Chem. Phys. 90, 1551 (1989).

${ }^{43}$ M. Braunstein and V. McKoy, J. Chem. Phys. 87, 224 (1987).

${ }^{44}$ M. Braunstein and V. McKoy, J. Chem. Phys. 90, 1535 (1989).

${ }^{45}$ R. E. Stratmann and R. R. Lucchese, J. Chem. Phys. 101, 9548 (1994).

${ }^{46}$ E. D. Poliakoff, M.-H. Ho, M. G. White, and G. E. Leroi, Chem. Phys. Lett. 130, 91 (1986).

${ }^{47}$ S. Kakar, E. D. Poliakoff, and R. A. Rosenberg, J. Chem. Phys. 96, 23 (1992).

${ }^{48}$ S. Kakar, H.-C. Choi, and E. D. Poliakoff, J. Chem. Phys. 97, 4690 (1992).

${ }^{49}$ J. S. Miller and E. D. Poliakoff, J. Chem. Phys. (in preparation).

${ }^{50}$ J. Kreile, H. D. Kurland, W. Seibel, and A. Schweig, Chem. Phys. 155, 99 (1991).

${ }^{51}$ C. Wu and E. D. Poliakoff, Chem. Phys. Lett. 292, 167 (1998). 